\title{
Aerogel RICH counter for the Belle II forward PID
}

Luka Šantelj $^{* a}$, I. Adachi' ${ }^{b}$, N. Hamada ${ }^{c}$, M. Higuchi' ${ }^{d}$, T. $\operatorname{lijima}^{e}$, S. Iwata ${ }^{f}$, H. Kakuno ${ }^{f}$, H. Kawai ${ }^{g}$, S. Korpar ${ }^{a h}$, P. Križan ${ }^{a i}$, S. Nishida ${ }^{b}$, S. Ogawa ${ }^{c}$, R. Pestotnik ${ }^{a}$,

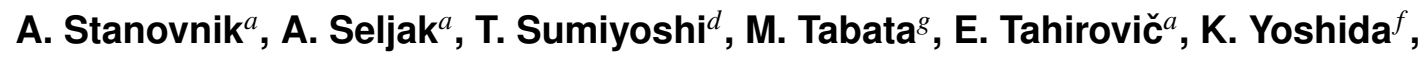
Y. Yusa ${ }^{j}$

a Jožef Stefan Institute, Ljubljana, Slovenia

${ }^{b}$ High Energy Accelerator Research Organization (KEK), Tsukuba, Japan

${ }^{c}$ Toho University, Japan

${ }^{d}$ Tokyo University of Science, Tokyo, Japan

e Nagoya University, Japan

${ }^{f}$ Tokyo Metropolitan University, Japan

${ }^{g}$ Chiba University, Japan

${ }^{h}$ University of Maribor, Slovenia

${ }^{i}$ University of Ljubljana, Slovenia

${ }^{j}$ Niigata University, Japan

E-mail: santelj.luka@ijs.si

The Belle II spectrometer, a follow up of the very successful Belle experiment, is under construction at the SuperKEKB electron-positron collider at KEK in Japan. For the PID system in the forward region of the spectrometer, a proximity focusing RICH counter with aerogel radiator is being developed. The detector will provide a $4 \sigma$ separation of pions and kaons up to momenta of $4 \mathrm{GeV} / c$, at the kinematic limits of the experiment. The main challenge in the development was a reliable multichannel sensor for single photons that operates in the high magnetic field of the spectrometer $(1.5 \mathrm{~T})$ and withstands the radiation levels expected at the experiment. A 144channel Hybrid Avalanche Photo-Detector (HAPD) was developed with Hamamatsu Photonics K.K. The design of the detector components is currently being finalized and part of the mass production have already started. The counter will be ready for installation in 2015. Here we report on the test of the prototypes conducted with test beam at DESY in 2013, and performance studies of the counter final design, based on the Geant 4 simulation.

Technology and Instrumentation in Particle Physics 2014,

2-6 June, 2014

Amsterdam, the Netherlands

${ }^{*}$ Speaker. 


\section{Introduction}

The Belle II experiment is a next generation $B$ physics experiment that will operate at the SuperKEKB asymmetric energy $e^{+} e^{-}$collider (Super $B$-factory) at KEK, Japan [1]. With an instant luminosity as high as $8 \times 10^{25} \mathrm{~cm}^{-2} \mathrm{~s}^{-1}$ SuperKEKB will open a window to a new panorama of measurements in heavy flavor physics, allowing high precision tests of the Standard model and searches for new physics effects through various (rare) decays of $B, D$ mesons and $\tau$ leptons. At the $B$-factory experiments a reliable particle identification (PID) is one of key elements. In particular, PID is crucial for tagging of $B$ meson flavor in the CP violation studies in neutral $B$ meson decays, and also for efficient background reduction in rare decays of $B$ and $D$ mesons. At the Belle experiment the PID of high momentum particles was provided by the Aerogel Cherenkov Counter (ACC), a threshold Cherenkov counter with aerogel as radiator, and fine mesh PMTs as photon detectors. However, for the upgraded Belle II experiment the ACC will be replaced by two new PID devices, time-of-propagation counter in the barrel and aerogel RICH counter (ARICH) in the endcap region [2]. In this letter we describe and review the status of the latter. The goal of the ARICH is mainly to improve and extend the very good $\pi / K$ separation to the full kinematic range of the experiment ( $4 \sigma$ separation at momenta between 0.5 and $3.5 \mathrm{GeV} / c$ ), and to provide discrimination between $\pi, \mu$ and $e$ below $1 \mathrm{GeV} / c$ (relevant for rare decays, such as $B^{0} \rightarrow K l l$ ).

The ARICH is a proximity focusing RICH with aerogel as a radiator [3]. The detector concept is shown in figure 1. Its main components are an aerogel radiator in which Cherenkov photons are emitted, an expansion volume to allow Cherenkov rings to expand, an array of position sensitive photon detectors, and a read-out system for the photon detectors. The main challenges that had to be meet by the detector design were very limited space $(\sim 28 \mathrm{~cm})$ and capability to operate in high magnetic field (1.5T) and harsh radiation environment (neutrons and gammas).

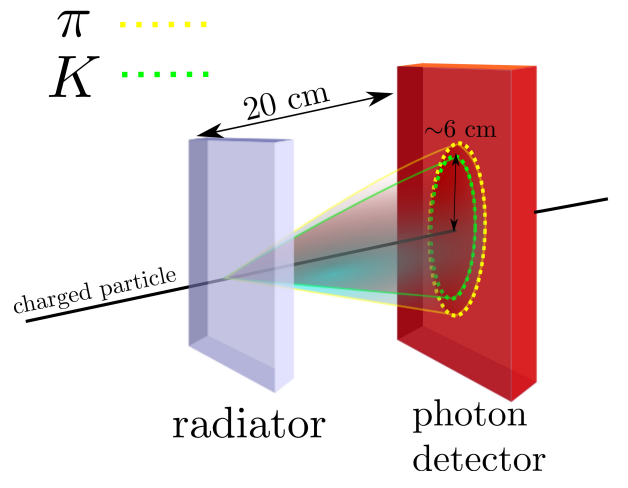

Figure 1: The concept of the ARICH.

\section{Detector Components and design}

\subsection{Radiator}

The key parameter in the performance of a RICH counter is the Cherenkov angle resolution per charged particle track, $\sigma_{\text {track }}=\sigma_{\theta_{C}} / \sqrt{N_{\text {det }}}$. With a longer radiator, the number of detected photons $\left(N_{\text {det }}\right)$ increases, but in a proximity focusing RICH the single photon angle resolution $\left(\sigma_{\theta_{C}}\right)$ degrades because of a larger emission point uncertainty. To at least partially overcome this difficulty the radiator plane of ARICH consists of two consecutive aerogel layers with high transparency $\left(\lambda_{T}>30 \mathrm{~mm}\right)$ and with different refractive indices [4]. By appropriately choosing their values, one can achieve overlapping of the corresponding two Cherenkov rings on the detector plane, which considerably reduces the spread due to emission point uncertainty. In our configuration the optimal 
thickness of each aerogel layer was found to be around $20 \mathrm{~mm}[5,6]$ and the refractive indices were chosen to be $n_{1}=1.045$ and $n_{2}=1.055$ [7]. With such a configuration approximately 10 photons per charged track, with $\sigma_{\theta_{C}}$ of about $13 \mathrm{mrad}$, are detected on the detector plane for hi-momentum particles. This is to be compared with a single, twice as thick, radiator layer where similar number of photons is detected but $\sigma_{\theta}$ of about $20 \mathrm{mrad}$ is observed [7].

In ARICH aerogel has to cover a large surface area, so it is also important to use large aerogel tiles in order to minimize the photon loss on the edges of tiles. We cover the detector surface with wedge shaped tiles, with approximate size of $18 \times 18 \mathrm{~cm}$, as shown in subsection 2.3 .

\subsection{Photon Detector}

As a photon detector a newly developed Hybrid Avalanche Photo Detector (HAPD) $[8,9]$ will be used (developed in joint effort of the Belle II collaboration and Hamamatsu Photonics). It provides high single photon detection efficiency with desired position resolution. The principle of HAPD operation and its picture are shown in figure 2. Incident photon is converted into photoelectron by a bi-alkali photo-cathode with peak quantum efficiency of $\sim 28 \%$ at $400 \mathrm{~nm}$. The electron is then accelerated in a vacuum tube with high electric field towards the segmented avalanche photo-diode with 144 pads of size $5.1 \times 5.1 \mathrm{~mm}$. Due to the avalanche gain of the photo-diode of about 40 and the bombardment gain of about 1700, the photon detection results in an avalanche of about 60000 electrons. For the readout a dedicated high gain and low noise electronics was developed: a digitizer ASIC which consists of a preamplifier, a shaper and a comparator (SA02) is followed by an FPGA (Xilinx Spartan-6 XC6SLX45), where the hit information is recorded and communicated to further stages of the experiment data acquisition [10].

One of the main prerequisites for the HAPD is its ability to operate in a harsh radiation environment. Our estimations, based on the simulation studies, show that neutron fluence at the place of HAPD positions can achieve $\sim 10^{12} \mathrm{n} / \mathrm{cm}^{2}$ in 10 years of the Belle II operation. Neutrons induce lattice defects in silicon bulk and leakage current in APDs increases, which results in lowering signal-to-noise ratio and therefore degradation of photon detection performance. During the development of HAPD several radiation tests were performed ${ }^{1}$, with neutron and gamma irradiation doses comparable and exceeding the expected values, resulting in several modification of APDs in order to minimize the radiation effects on their performance ${ }^{2}$. By introducing this modifications HAPD now satisfies our requirements of radiation hardness for long term operation [11].

\subsection{Design}

In total ARICH consists of 420 HAPD modules arranged in seven concentric rings $\left(r_{\text {in }}=56\right.$ $\mathrm{cm}, r_{\text {out }}=114 \mathrm{~cm}$ ) and of $2 \times 124$ aerogel tiles of wedge shape, as shown in the left and middle subfigure of figure 3, respectively, where one of the six detector sectors is shown. In order to maintain good performance also on the outer edge of the detector, where Cherenkov photons would miss the photo-sensitive area, 18 planar mirror plates are placed as shown and sketched in the middle and right subfigure of figure 3.

\footnotetext{
${ }^{1}$ Tests were performed in a neutron beam line at the JPARC facility $\left(\sim 10^{12} \mathrm{n} / \mathrm{cm}^{2}\right)$ and at the ${ }^{60}$ Co irradiation facility at Nagoya University (up to 1000 Gy).

${ }^{2}$ Reduction of thickness of the $p$ and $p+$ layers, optimization of the protective layers, placing of an additional intermediate electrode.
} 

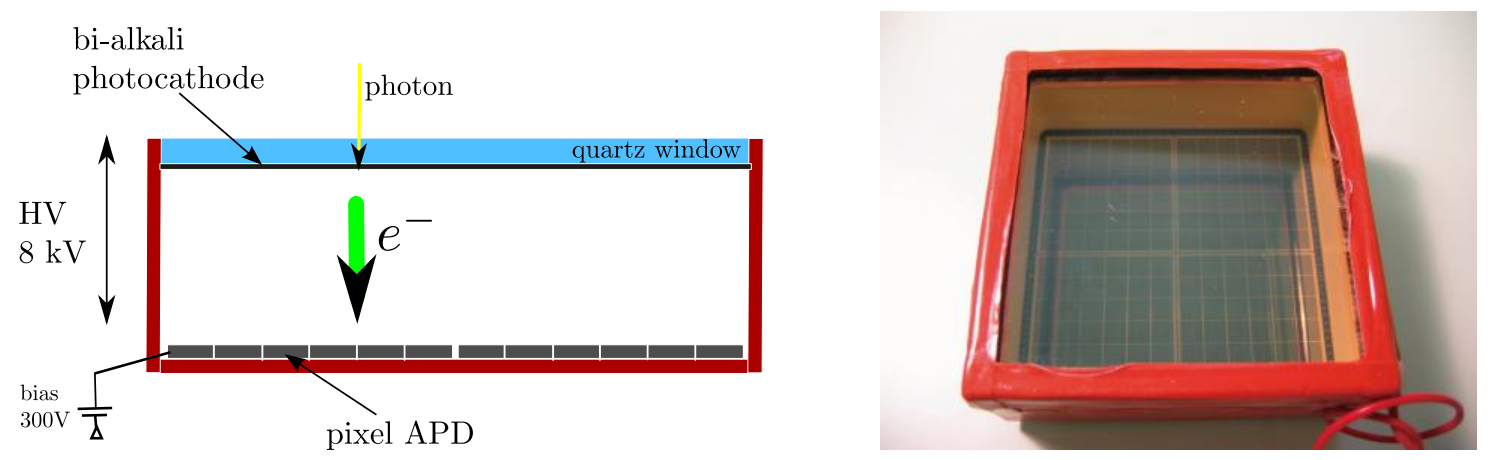

Figure 2: Left: Principle of HAPD operation. Right: Photograph of HAPD module.
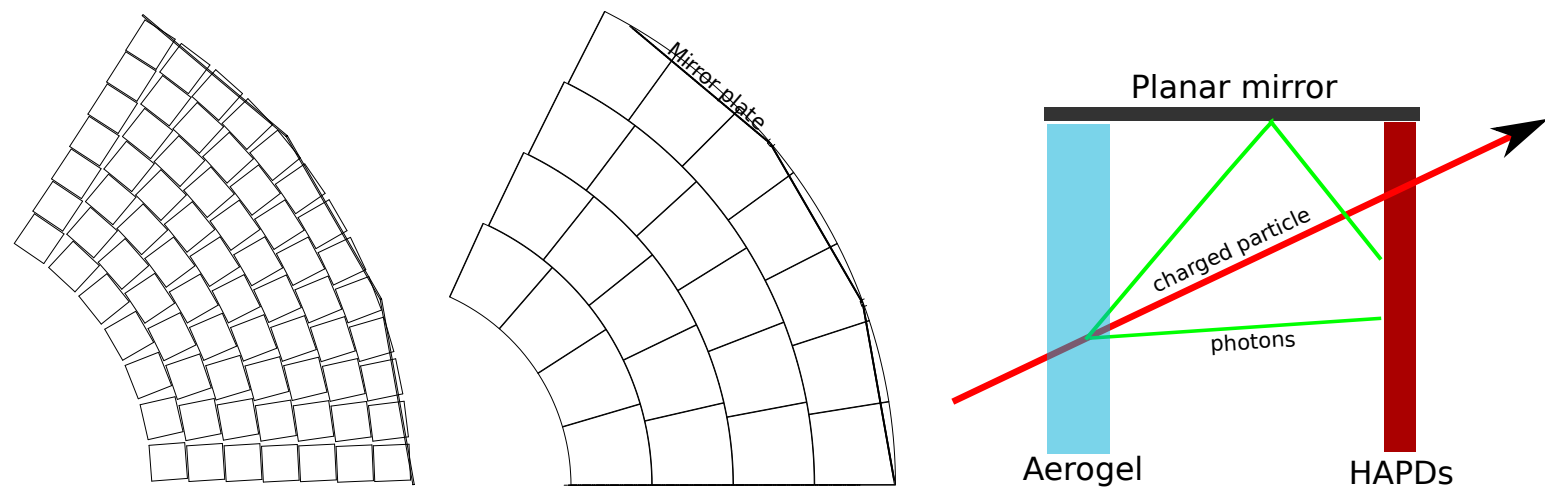

Figure 3: Left: Distribution of HAPD modules over one sixth of the detector plane. Middle: Layout of the aerogel tiles configuration. Right: The effect of planar mirrors on the outer side of ARICH.

\section{ARICH performance in simulation studies}

To optimize the ARICH parameters and test its performance a detailed Geant 4 simulation of the detector was developed. On figure 4 an event example from the simulation is shown (left), along with the distributions of reconstructed Cherenkov angle (middle) and calculated $\pi / K$ hypothesis likelihood difference (right) for pions and kaons with momenta at $3.5 \mathrm{GeV}$. Particles are shoot from the interaction point and the information of particle track position and direction on the aerogel plane (needed for $\theta_{C}$ reconstruction) is taken from the simulation and smeared for the expected resolution of the Belle II tracking system. Two well separated peaks in the likelihood difference distributions (figure 4 right) demonstrate excellent $\pi / K$ separation ability of the detector, exceeding $99 \%$ efficiency for kaon identification at $2 \%$ pion misidentification probability.

The developed Geant4 description of ARICH geometry is also integrated into the Belle II full detector simulation, which is used for Belle II performance studies and production of simulated event data, needed for measured data analyses. Therefore we also study the ARICH performance using the full Belle II simulation, where instead of the true track information (from simulation), reconstructed information from the Belle II tracking system is used (drift chamber + vertex de- 

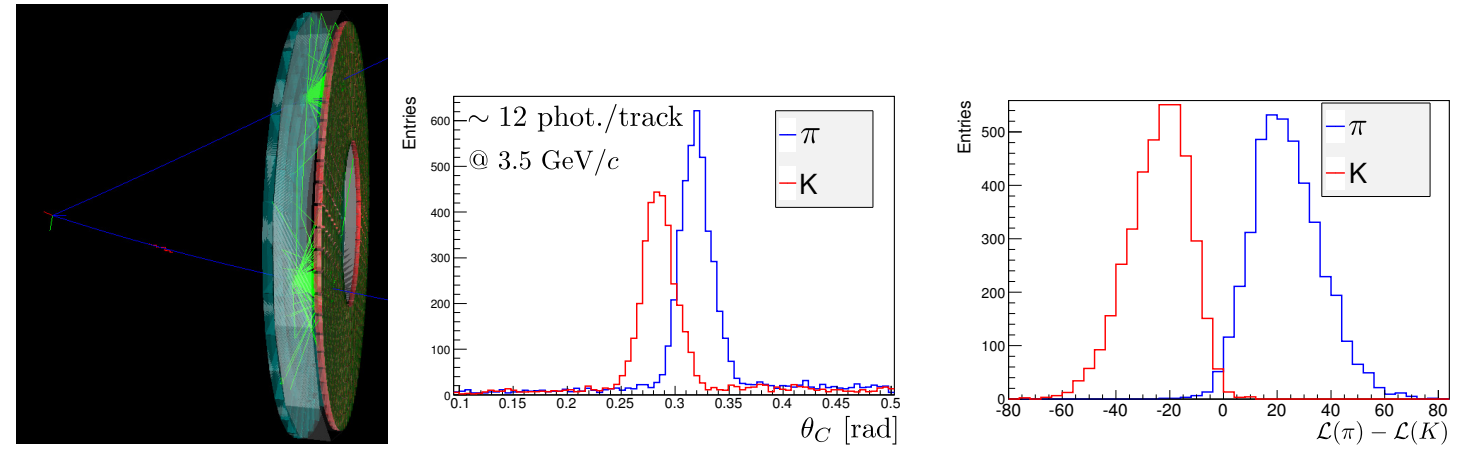

Figure 4: Left: An example event from Geant4 simulation of ARICH. Middle: Distributions of reconstructed Cherenkov angle for $\pi$ and $K$ tracks with momenta $3.5 \mathrm{GeV} / c$. Right: Corresponding distributions of calculated $\pi / K$ hypothesis likelihood difference for $\pi$ and $K$ tracks.

tectors). We simulate single $\pi / K$ track events with particle momenta ranging from $0.5 \mathrm{GeV} / c$ to $4.0 \mathrm{GeV} / c$, as well as $e^{+} e^{-} \rightarrow \Upsilon(4 S) \rightarrow B^{0} \bar{B}^{0}$ events, with one $B^{0}$ decaying generically and one as $B^{0} \rightarrow K \pi$. Main performance results are shown in figure 5. Due to a small fraction of badly reconstructed tracks we observe a slight decrease in $K$ identification efficiency compared to the previously mentioned ARICH stand-alone study. For tracks from $B^{0} \rightarrow K \pi$ decay (their momenta are in a range $3-3.5 \mathrm{GeV} / \mathrm{c}$ ) we correctly identify $98 \%$ of kaons while misidentifying $2 \%$ of pions.
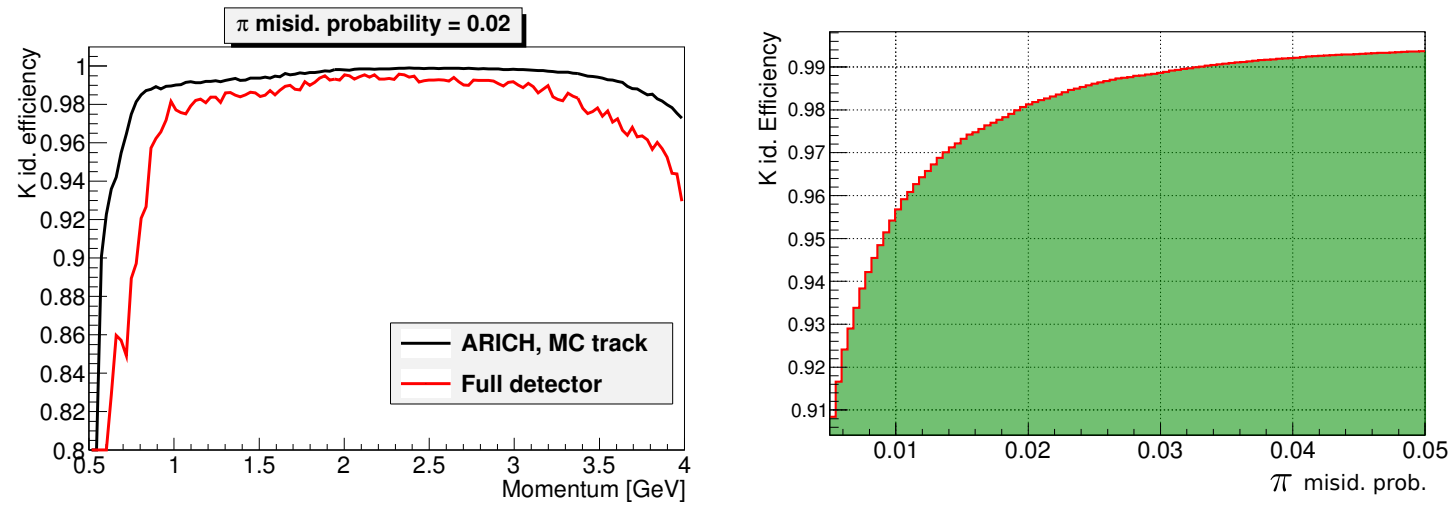

Figure 5: Left: $K$ identification efficiency at $2 \% \pi$ misidentification probability as a function of particle momentum. The black and the red line show results from the ARICH stand-alone study (track parameters from MC) and from the full Belle II simulation (track parameters from the tracking system). Right: $K$ identification efficiency as a function of $\pi$ misidentification probability for $K$ tracks arising from the $B^{0} \rightarrow K \pi$ decay.

\section{ARICH performance in test beam measurements}

To test the performance of the designed ARICH we constructed a small prototype, with two consecutive aerogel tiles and six HAPD modules, arranged as in a part of actual detector layout. In the recent few years we have performed three beam tests, at KEK in 2009 [9] $3 \mathrm{GeV} / c$ electron beam), at CERN in 2011 (120 GeV/c hadron beam), and at DESY in 2013 (4-5 GeV/c electron 
beam). Here we report on some results from the latest test. The sketch and photograph of the experimental setup are shown in figure 6. In some of test beam runs a mirror was placed at the side of detectors, as can be seen on the photograph, and the full setup was rotated for different angles with respect to the beam direction. The detector prototype was enclosed in a light shielding box, four multi-wire proportional chambers were installed (two in front and two after the box) to provide charged particle track information, and the trigger signal was provided by two plastic scintillator counters.
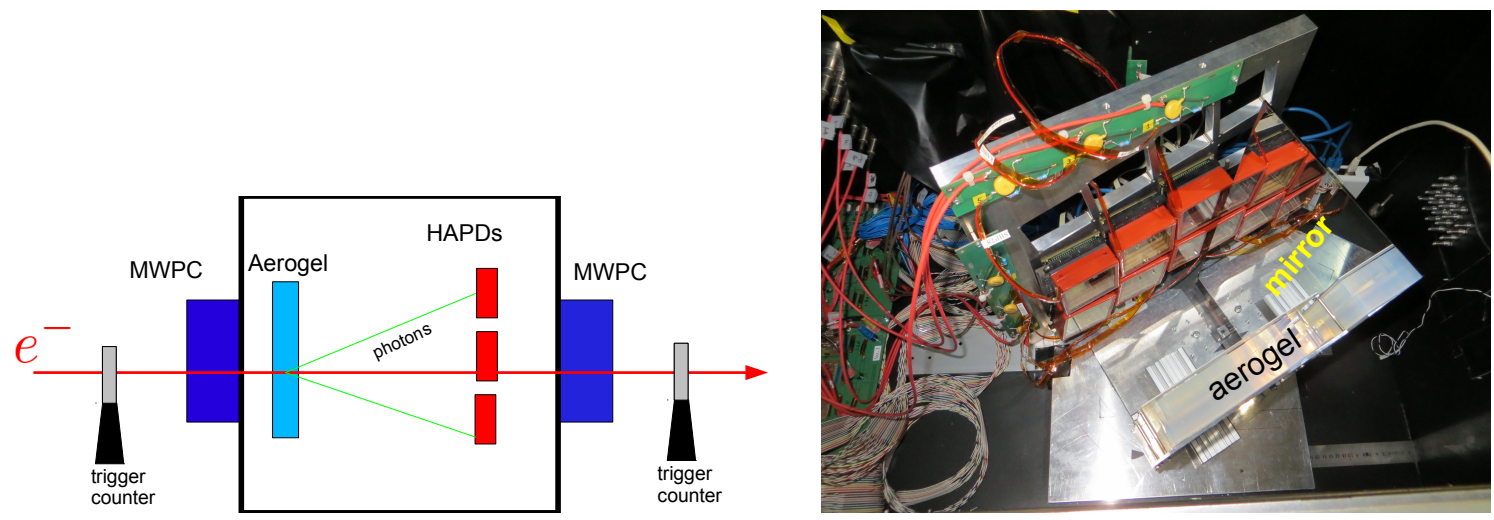

Figure 6: Sketch and photograph of the ARICH prototype experimental setup used in test beam at DESY, 2013.

In figure 7 accumulated distribution of the reconstructed Cherenkov photon emission angle is shown in two dimensional Cherenkov space (left) and its projection on the $\theta$ angle (right), for the tracks with zero incidence angle. We fit the data distribution with a sum of Gaussian and linear functions and obtain the width of the Cherenkov peak $\left(\sigma_{\theta_{C}}\right)$ of about $12.8 \mathrm{mrad}$. The average number of detected photons per track $\left(N_{\text {det }}\right)$ is about 9 . From this information a "naive" estimate of $\pi / K$ separation power can be obtained using $\Delta \theta_{C} /\left(\sigma_{\theta_{C}} \sqrt{N_{\text {det }}}\right)$, where $\Delta \theta_{C}$ is about $23 \mathrm{mrad}$ for $\pi / K$ tracks at $4 \mathrm{GeV} / c$. Inserting the numbers we obtain separation of about $5.5 \sigma$, satisfying and exceeding our performance criteria. We also prepare a Geant 4 simulation of the described experimental setup and compare the results with measured data. The distribution of reconstructed Cherenkov angle for both cases is shown in left subfigure of figure 8. We find a very good agreement in the single photon angle resolution and the number of detected photons per track, but the amount of background photons is underestimated in the simulation. This is mainly due to multiple track events in the measured data and instrumental noise, which are not included in the simulation.

As already mentioned, we also tested the performance with placed mirror and rotated setup. For example, on the right subfigure of figure 8 we show the reconstructed Cherenkov ring that is fully reflected by the mirror. Fit of the reconstructed $\theta_{C}$ distribution gives the Cherenkov peak width of about $11.6 \mathrm{mrad}$, exceeding the resolution of non-reflected rings. This is expected due to the longer photon travel distance. 

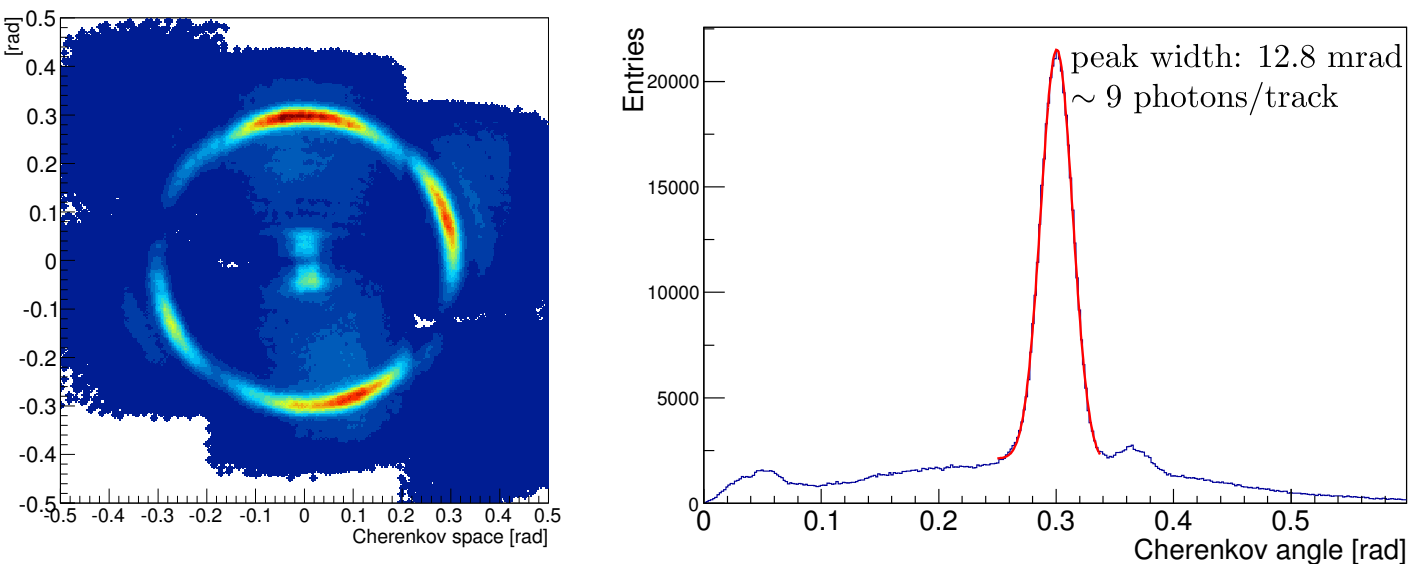

Figure 7: Left: Accumulated distribution of reconstructed Cherenkov photon emission angle in the Cherenkov space (i.e. Cherenkov ring). Right: The blue line shows the accumulated distribution of reconstructed $\theta_{C}$ angle and the red line shows the fit result.
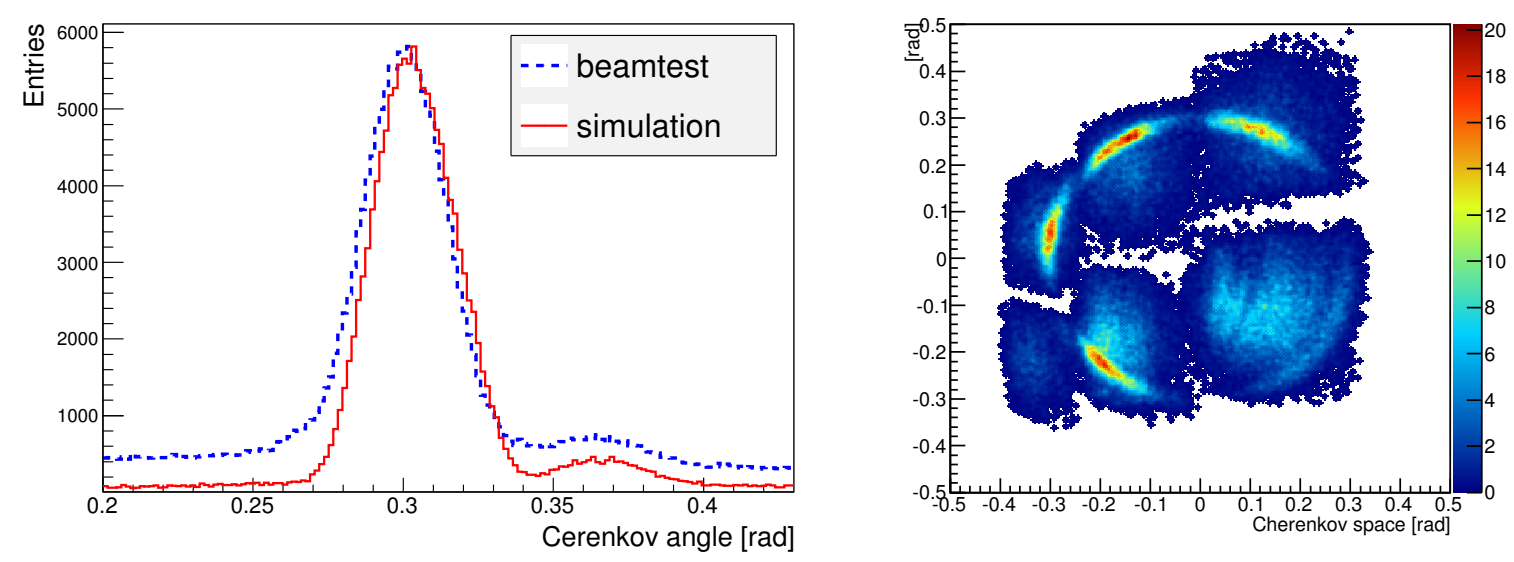

Figure 8: Left: Comparison of the accumulated distributions of reconstructed Cherenkov photon emission angle from the simulation and from the measured data (non-normalized; peak heights are set to be equal for easier peak position and width comparison). Right: Reconstructed Cherenkov ring reflected by the mirror.

\section{Summary and schedule}

For the upcoming Belle II experiment we are constructing an aerogel RICH counter (ARICH) that will provide particle identification in forward endcap region of the detector. Its main objective is very good $(>4 \sigma)$ separation between pions and kaons in the full kinematic range of the experiment $(0.5-3.5 \mathrm{GeV} / c)$ and sufficient discrimination between low energy $(<1 \mathrm{GeV} / c) \pi, \mu$ and $e$. The counter design was guided by the very limited available space, performance in high magnetic field, and high radiation tolerance of photon detectors. ARICH consists of two aerogel layers in a so-called focusing configuration $\left(n_{1}=1.045, n_{2}=1.055\right)$, an array of 144-channel Hybrid Avalanche Photo-Detectors (HAPDs), and dedicated readout electronics based on ASIC. We have 
confirmed the desired performance of the designed ARICH in a detailed Geant 4 simulation studies, as well as by constructing a small prototype detector and test it in several test beams. In these tests we achieved about $5.5 \sigma$ separation between $\pi / K$ Cherenkov rings, satisfying and exceeding performance requirements. Most of the detector components are already in the mass production. So far about 250 HAPD were produced, about 200 were already tested, and 150 are qualified as good samples, while some are still under investigation (noisy or large leakage current). The production is to be finished around March 2015. The aerogel tiles were already produced and measurements of their optical properties are ongoing. Final version of readout electronics is now being tested and mass production is planned to begin in autumn. In early 2015 the ARICH is to be constructed and its installation is planned for summer 2015.

\section{References}

[1] T. Abe et al., arXiv:1011.0352 [physics.ins-det]

[2] R. Pestotnik et al., Nucl. Instrum. Meth. A 732 (2013) 371-374.

[3] T. Iijima et al., Nucl. Instr. and Meth. A 453 (2000) 321; E. Nakano, Nucl. Instr. and Meth. A 494 (2002) 402.

[4] M. Tabata et al., arXiv:1406.4564 [physics.ins-det]

[5] T. Matsumoto, S. Korpar et al., Nucl. Instr. and Meth. A 521 (2004) 367.

[6] T. Iijima, S. Korpar et al., Nucl. Instr. and Meth. A 548 (2005) 383; S. Korpar et al., Nucl. Instr. and Meth. A 553 (2005) 64.

[7] S. Korpar et al., Nucl. Instrum. Meth. A 572 (2007) 429.

[8] S. Nishida et al., Nucl. Instr. and Meth. A 595 (2008) 150.

[9] I. Adachi et al., Nucl. Instr. and Meth. A 623 (2010) 285.

[10] S. Nishida et al., Nucl. Instrum. Meth. A 623 (2010) 504; A. Seljak et al., JINST 6 (2011) C01083;

[11] S. Korpar et al., Proceedings of RICH2013, to be published in Nucl. Instrum. Meth. A. 